\title{
EDITORIAL OPEN Superconducting quantum bits with artificial damping tackle the many body problem
}

npj Quantum Information (2019)5:21; https://doi. org/10.1038/s41534-019-0135-1

C ircuit QED represents one of the most advanced architectures in the race for a quantum computer. Its elementary building blocks, the superconducting quantum bits and microwave cavities, play the role of nearly ideal spins and springs. Thanks to technological progress, the spin/spring coupling constant greatly exceeds the decoherence rate of the system, so that spins and springs get strongly quantum-entangled. This strong coupling regime is exploited by numerous teams to perform quantum algorithms with a growing number of quantum bits. ${ }^{1}$ In this context, dissipation sources, which cause decoherence, appear as the physicist enemy number one, which limits the size of quantum computers and their performances. A lot of efforts is devoted to the development of quantum error correction codes which fight the destruction of quantum information. ${ }^{2}$ This requires to increase the number of physical bits used to encode one bit of information, making the realization of complex quantum algorithms even more challenging.

However, a weakness can sometimes be turned into a strength. Indeed, superconducting circuits can also be used to perform a direct simulation of intrinsically dissipative quantum systems, in principle. In this case, decoherence in the circuit must mimic the one in the real system, and it thus turns from a spurious effect to a key ingredient. For example, one can imagine to emulate in a controlled way the Kondo effect, which epitomizes many body condensed matter problems. This effect, which was discovered in the 30 's, consists in a low temperature increase of the resistance of some metals, due to the spin-flip scattering of their continuum of itinerant electrons on magnetic impurities. ${ }^{3}$ The Kondo model is also central to understand strongly correlated electron behavior in quantum dot circuits, heavy-fermion materials and hightemperature superconductors. ${ }^{4}$ It is possible to make an exact theoretical description of the Kondo effect in some limiting cases like the high or low temperature regimes, but the general case remains challenging. Theorists expect the formation of an intriguing Kondo cloud of itinerant electrons, with a spin entangled with impurity spins, but this feature could not be observed directly so far. ${ }^{5}$

Recently, it has been suggested to simulate Kondo physics with photons by using a superconducting quantum bit coupled to two semi-infinite microwave transmission lines. ${ }^{6,7}$ This physical situation corresponds to a dissipative spin/boson model, where dissipation is provided by the transmission lines due to their continuous density of photonic modes. Kondo physics is expected because there exists a direct mapping between the dissipative spin/boson model and the Kondo model..$^{8}$ Nevertheless, this geometry cannot fully exploit the power of Circuit QED. Indeed, microwave resonant techniques, which enable an efficient control and monitoring of quantum bits, cannot be used to reveal directly the phenomena which occur inside a photonic continuum. Yet...it is possible to sit on the fence by using a very long but finite waveguide. In this case, the waveguide forms a cavity with a discrete spectrum, but the round-trip of photons in this cavity is much longer than the qubit/cavity mode coupling constant, so that the qubit effectively feels an "artificial" dissipative environment, at least for times shorter than the photonic round trip. If, on top of that, the qubit/cavity coupling is stronger than the energy spacing between the photonic modes, and the qubit nonlinearity sufficiently large, many-body physics is expected. Strinkingly, in this geometry, the many modes of the effective bath could be addressed individually thanks to microwave resonant techniques. One can thus dream of measuring directly the Kondo cloud, or even its formation and dynamics in quench experiments where the system would be brought suddenly into the Kondo regime.

Motivated by these perspectives, several teams have revisited experimentally the problem of a superconducting qubit coupled to a long microwave coplanar waveguide., ${ }^{9,10}$ Now, these experiments are brought to the next technical level by two groups who use arrays of Josephson junctions ${ }^{12}$ or squids ${ }^{11}$ as a waveguide. These arrays have a high impedance that favors strong qubit/bath couplings. Furthermore, in ref. ${ }^{11}$, the squid array architecture enables a strong tunability of the bath modes with the magnetic field, which could be instrumental to study the parametric dependences in a many-body problem. Puertas-Martínez and coworkers ${ }^{11}$ perform an impressively accurate parameter-free modeling of their experiment, which reveals the breakdown of the rotating wave approximation, expected in the multimode strong coupling regime. Kuzmin and colleagues ${ }^{12}$ obtain an even larger qubit/bath coupling, so that individual anticrossings between the qubit and the numerous bath modes cannot be distinguished anymore due to the complete dissolution of the qubit state in these modes. In both experiments, the bath levels are resolved and studied individually. In order to enter the deep many-body regime, further experimental efforts are necessary to increase the qubit nonlinearity. However, considering the rapid experimental progress, one can hope the simulation of many-body physics with superconducting circuits to be within reach soon.

\section{ADDITIONAL INFORMATION}

Competing interests: The author declares no competing interests.

Publisher's note: Springer Nature remains neutral with regard to jurisdictional claims in published maps and institutional affiliations.

Audrey Cottet $^{1}$

${ }^{1}$ Laboratoire de Physique de l'Ecole normale supérieure, ENS, Université PSL, CNRS, Sorbonne Université, Université Paris-Diderot, Sorbonne Paris Cité, Paris, France Correspondence: Audrey Cottet (cottet@lpa.ens.fr)

\section{REFERENCES}

1. Wendin, G. Quantum information processing with superconducting circuits: a review. Rep. Prog. Phys. 80, 106001 (2017).

2. Devoret, M. H. \& Schoelkopf, J. R. Superconducting circuits for quantum information: an outlook. Science 339, 1169 (2013). 
3. Kondo, J. Resistance minimum in dilute magnetic alloys. Progress. Theor. Phys. 32, 37 (1964).

4. Kouwenhoven, L. \& Glazman, L. Revival of the Kondo effect. Phys. World 14, 33 (2001).

5. Desjardins, M. M. et al. Observation of the frozen charge of a Kondo resonance. Nature 545, 71 (2017)

6. Le Hur, K. Kondo resonance of a microwave photon. Phys. Rev. B 85, 140506 (2012). (R).

7. Goldstein, M., Devoret, M. H., Houzet, M. \& Glazman, L. I. Inelastic microwave photon scattering off a quantum impurity in a josephson-junction array. Phys. Rev. Lett. 110, 017002 (2013).

8. Blume, M., Emery, V. J. \& Luther, A. Spin-boson systems: one-dimensional equivalents and the kondo problem. Phys. Rev. Lett. 25, 450 (1970).

9. Forn-Díaz, P. et al. Ultrastrong coupling of a single artificial atom to an electromagnetic continuum in the nonperturbative regime. Nat. Phys. 13, 39 (2017)

10. Sundaresan, N. M. et al. Beyond strong coupling in a multimode cavity. Phys. Rev. $X$ 5, 021035 (2015).

11. Puertas-Martínez, J. et al. A tunable Josephson platform to explore many-body quantum optics in circuit-QED. npj. Quant. Inf. 5, 19 (2019).
12. Kuzmin, R. et al. Superstrong coupling in circuit quantum electrodynamics. npj. Quant. Inf. 5, 20 (2019).

(c) Open Access This article is licensed under a Creative Commons Attribution 4.0 International License, which permits use, sharing, adaptation, distribution and reproduction in any medium or format, as long as you give appropriate credit to the original author(s) and the source, provide a link to the Creative Commons license, and indicate if changes were made. The images or other third party material in this article are included in the article's Creative Commons license, unless indicated otherwise in a credit line to the material. If material is not included in the article's Creative Commons license and your intended use is not permitted by statutory regulation or exceeds the permitted use, you will need to obtain permission directly from the copyright holder. To view a copy of this license, visit http://creativecommons. org/licenses/by/4.0/.

(c) The Author(s) 2019 